\title{
Research on Fragment Reading in Mobile Internet
}

\author{
Ruixiang $\mathrm{Ou}^{1}$, Hui $\mathrm{Pan}^{1}$, Yao Huang ${ }^{2}$ \\ ${ }^{1}$ South China University of Technology Library, Guangzhou, 510640, China \\ ${ }^{2}$ Department of Propaganda, Party Committee, Southern Medical University, \\ Guangzhou 510515, China
}

Key words: Mobile internet; fragmented reading; reading mode

Abstract: Reading is an important part of people's social life. With the accelerated pace of life, people retain the traditional reading habits and methods, but also began to get used to fragmented reading. This paper studies the manifestation and characteristics of fragmented reading in the mobile Internet, analyzes the problems of fragmented reading in the mobile Internet, and puts forward the views that we should regard fragmented reading in scientific and rational way.

The fragmented reading of the mobile Internet era is "a three-dimensional way of acquiring knowledge and information", it includes not only the traditional text reading, but also the sound, images and other symbolic content reading by network and media technology. This reading method included three aspects of reading, listening, reading compared with the traditional reading, which is more three-dimensional and modern . Fragmentation reading does not make reading carrier and reading time as a definition standard, but highlight the fragmentation of reading content; this fragmented reading mode in the mobile Internet era is more obvious.

\section{The manifestation of fragmented reading in mobile Internet age}

\section{(1) The reading time is fragmented}

With the development of economy, time and attention have become scarce resources for social members to enhance themselves, which forces people to divide time into pieces and receive valid information in fragmented times. People can read in fragmented time, which make the limited time become more effective and improve the value of time by the modern media-assisted network. The fragmented reading can effectively use the characteristics of the media to make the traditional reading time suit the fragmented mobile Internet life.

\section{(2) The reading content is fragmented}

With rapid development of media technology in the mobile Internet, the diversified development of the media provides more and more information, the information becomes more efficient, short and rich. These characteristics of the information have led to the formation of fragmented reading thinking. The fragmentation of reading content is mainly manifested in the complexity of reading content.

\section{(3) The reading forms are fragmented}

Fragmentation read is mainly through a variety of information receiving equipment to complete the reading activities. In mobile Internet age, people can send or receive information anytime and anywhere and interact with others by mobile phones, tablet PCs. People can read with the fast browsing, selective 
reading, skip reading, making reading showing fragmentation, dynamic and instantaneous and other characteristics. This way help people get rid of time and space constraints of the traditional reading, free to read anytime and anywhere.

\section{(4) The reading logics are fragmented}

The fragmented reading in the mobile Internet age is not linear, single, stable, and the reader can choose the content according to his own needs and interests. Reading time fragmentation allows the reader to relax in the entire reading process, which is a more casual reading, with a strong random and entertainment tendency. Reading content fragmentation allows readers to read the contents which are relatively shallow; this reading has a strong jump and browsing. So the reader in the process of reading is difficult to form a rigorous logical analysis.

\section{The features of fragmented reading in mobile Internet age}

(1) Interactivity

The interaction of fragmented reading in the mobile Internet era is mainly manifested in three aspects (1) the interaction between the reader and the author,(2) the interaction between the reader and the reading text, (3) the interaction between the reader and the reading medium. In the context of mobile Internet fragmentation, the author and the reader are blurred, and it can use the network for real-time interaction and communication. This interaction makes the reader no longer accept the information passively, but actively create the content and make the fusion of identity.

\section{(2) Real-time and mobility}

Mobile Internet era is the age of rapid development of large data. The hypertext link function of the network not only provides massive information for people, but also connects numerous pieces of information with large data. Mobile Internet support the use of multi-window operation with various types of mobile terminal equipment, the presentation of information is no longer limited to the text, but make the picture, video and sound into the information content. This large data makes people's reading anytime and anywhere, you can freely switch content that you interested in.

\section{(3) Propagation of non-linearity}

The way of information organization in Mobile Internet is non-linear way, it reorganizes a variety of information resources to make immediate dissemination, real-time interaction through the Internet technology. The Internet links the information resources of different locations and different events in the same text, making the information appear in a mesh structure. This reorganization is not limited to traditional reading in the simple text information, but also added a wealth of sound, pictures and video content. Fragmentation readings help people develop the ability to read and diverge thinking, which in turn will promote the reader's ability to understand and the formation of imagination.

\section{(4) Acceptable accumulation}

In the process of reading, the reader will link the new information with the original information to build a new information network in subconscious. The 
rapid accumulation of information is the most obvious feature of fragmented reading, because the content of fragmented reading is easy to accept, and it is easy to spread. The richness of information can make readers access to different areas of knowledge, and this long-term accumulation can also enhance people's understanding of things. The problem of deep reading of fragment reading can be overcome through the accumulation of information. Readers use fragmented time to make fragmented reading, continue to accumulate the reading amount and join the reader's knowledge system, which become an important source of knowledge for the future of the cognitive world.

\section{The problems of fragmented reading in mobile Internet age}

\section{(1) Excessive reliance on bad pieces of information leads to self-loss}

Excessive fragmented reading will, to a certain extent, make the reader dependent on the media, is accustomed to obtaining information fragments by means of search or interaction rather than conducting independent thinking, and fragmented information in the reader's brain cannot create an integral knowledge of the map.

In addition, the process of fragmented reading is often disturbed by information that is irrelevant to the reading subject, but is turned to the content by a curious or time problem, so that it will deviate from the original target reading. That is, fragmented reading there is easy to jump from the target reading to blurred reading embarrassing situation. So that many different information in the constant Free State, the amount of information is easy to exceed the reader's information load. At the same time with the network search retrieval, it not only will not bring convenience, and even cost us a lot of time and effort, and further intensify the fragmentation in the network society lost, so the formation of reading logic thinking ability will inevitably not be debris, traditional reading is aimed at acquiring knowledge by reading information to sublimate wisdom, which is indispensable for fragmented reading.

\section{(2) Accept a lot of debris information quickly leads to distraction}

Internet media in Mobile Internet age has gradually developed into the most powerful information aggregator, whether it is political, economic, cultural or entertainment information, people want to know the first thing in the first time , the Internet and mobile terminals are well satisfied people's knowledge needs. But in the process of reading the massive fragmented information, the reading speed is accelerating, the reading content is increasing, and the reader's attention is constantly distracted.

The result of this distraction is not only the impact of long reading and deep reading, but also it will bring this fragmented consciousness into people's lives, learning and work. That is, the adverse effects of fragmented reading is not limited to the field of reading, but also spread to other aspects of life.

\section{(3) Access shallow information led to thinking ability decreased}

People use media and network technology to effectively retrieve the complex information. This long fragmented reading makes people read and understand ability of large or long article decline; this reading mode is likely to cause reading 
thinking of the inertia, which will further affect people's way of thinking, rational thinking ability, logical thinking ability and ability to judge. On the other hand, one of the main features of fragmented reading is the lack of access to a large amount of information in a short period of time, the variety of information and the speed of updating result in the lack of deep and sustained attention to the understanding of certain information. The fragmented reading is not able to achieve the depth of reading excellent books, but also cannot achieve the depth of participation in the reading process.

\section{(4) Reading motives have a tendency to entertain}

With the development of the media and the depth of mobile phone reading, no time to read, reading fragmentation, entertainment and utilitarian tend to become increasingly obvious, which has become a common phenomenon in today's society. The contents of the fragmented reading are mostly entertainment news and entertainment gossip and other words alone, rather than the traditional sense of reading the academic knowledge. But the content of fragmented reading is mostly the focus of attention in daily life. The value of fragmented reading content cannot be compared with the traditional classical literature. Excessive reading of fragmented information will only paralyze the nerves that people think about, prompting people to stay in the superficial observation rather than to think deeply.

The proliferation of fragmented reading is likely to deprive readers of the ability to perform traditional deep reading. Some texts in order to meet the reader's novelty and entertainment psychology lead some reader who is easy to read in the lost self. Because of the fragmented characteristics of the network culture, the reading content of the people in which the reading is obtained is empty, and the whole reading process is superficial. The whole environment of the fragmented reading is impetuous and will lead to the "reading crisis".

\section{Make rational view of fragmented reading in mobile Internet age}

(1) Face the way of reading mode changes

The emergence of fragmented reading is the inevitable result which can adapt to social development, meet the people read of the demand. With accelerating the pace of living, this new way of life makes people fragmented time increases. How to use effectively the split time becomes the most concerned problem. We should follow the development of the times, change the existing reading ideas, to create a good reading environment, better enhance the quality of individual and make contributions for social and cultural progress.

\section{(2) Make rational view of the advantages and disadvantages of reading, improve reading literacy.}

Although the fragmented reading of the mobile Internet era did not set a higher reading threshold for the reader, but still need to have a certain communication quality and communication ability to enhance their media literacy, it not simply meet the adventures rational to read, While only in this way, the process of fragmented reading can achieve self-improvement.

From the point of view of the communicator, the fragmentation information can 
be used to refine the effective information to facilitate the dissemination of the most valuable information content, so that the information disseminated is fragmented and holistic. From the perspective of the audience, the mobile Internet era requires the audience to have a relatively complete knowledge structure and a high logical thinking ability; in the fragmented reading process reader can be independent thinking.

(3) Make traditional Reading and fragmentation Reading in scientific balance

Learn respective values from the traditional reading and fragment reading, that is, on the basis of moderate fragmentary reading, to encourage the traditional depth of reading, thinking and reflection, so as to enhance the level of cultural reading. Government and the publishing agencies need to create a good reading environment, increase investment in the development of cultural industries, innovative reading platform in mobile Internet era.

We only make the fragmented reading in-depth study and excavation, maintain scientific awareness of fragmented reading and make reasonable use of the value of reading, it can guide cultural well develop and promote the progress of civilization.

\section{Acknowledgement}

This paper is one of the research results of the Influence of Fragment Reading on College Students' Literature Reading Appreciation in micro-propagation environment (Project No: GD15XTS06), which is the "Twelfth Five - Year Plan" of Guangdong Province 's Philosophy and Social Science in 2015.

\section{References}

[1] Xu Yan. An Empirical Study on Fragment Reading Behavior Based on Information Literacy - A Case Study of Library WeChat Platform [J]. Information Science. 2017 (03): 76-81.

[2] ZHAO Shu-hai.Study on the study of fragmented network reading behavior of college students and its influencing factors [J]. Library Science Research. 2016 (22): 61-63.

[3] Wang Yanling. Let fragmented reading become a classic guide to the effective way [J]. News lovers. 2015 (03): 66-68.

[4] Dong Lili. Analysis of the advantages and disadvantages of fragmented reading from the media age [J]. Published wide angle 2015 (12): 110-111.

[5] Chen Yi, Ling Meng Dan. Microblogging "fragmented reading" of the spread of anesthesia function interpretation [J]. Editor of the Friends. 2014 (05): 19-21. 